EESTI NSV TEADUSTE AKADEEMIA TOIMETISED. XIII KÖIDE

FOOSIKA-MATEMAATIKA-JA TEHNIKATEADUSTE SEERIA. 1964, NR. 2

ИЗВЕСТИЯ АКАДЕМИИ НАУК ЭСТОНСКОИ ССР. ТОМ ХІІІ

СЕРИЯ ФИЗИКО-МАТЕМАТИЧЕСКИХ И ТЕХНИЧЕСКИХ НАУК. 1964, № 2

\title{
О ВЗАИМОДЕЙСТВИИ ТРИИЗОБУТИЛАЛЮМИНИЯ С АЛКИЛХЛОРИДАМИ
}

\author{
Х. КААР, Г. ШВИНДЛЕРМАН
}

В работах Позамантира и Генусова [1, 2] было показано, что скорость взаимодействия алкилгалогенидов с $\mathrm{R}_{3} \mathrm{Al}$ возрастает по мере увеличения положительного индуктивного эффекта в ряду: $\mathrm{C}_{2} \mathrm{H}_{5} \mathrm{Cl}<n-\mathrm{C}_{4} \mathrm{H}_{9} \mathrm{Cl}<$ изо- $\mathrm{C}_{3} \mathrm{H}_{7} \mathrm{Cl}<$ трет- $\mathrm{C}_{5} \mathrm{H}_{11} \mathrm{Cl}$.

Реакция представляет собой последовательное замещение алкильных групп прн Al на галоид: $\mathrm{R}_{3} \mathrm{Al} \stackrel{\mathrm{R}^{\prime} \mathrm{X}}{\longrightarrow} \mathrm{R}_{2} \mathrm{AlX} \stackrel{\mathrm{R}^{\prime} \mathrm{X}}{\longrightarrow} \mathrm{RAlX}_{2} \stackrel{\mathrm{R}^{\prime} \mathrm{X}}{\longrightarrow} \mathrm{AlX}_{3}$, причем при переходе от $\mathrm{R}_{3} \mathrm{Al}$ ж $\mathrm{RAlX}_{2}$, скорость реакции увеличивается. Taк, $\mathrm{R}_{2} \mathrm{AlCl}$ быстро реагирует с алифатическими галоидалкилами, у которых имеется более двух атомов углерода $\left[{ }^{3}\right]$, и даже с хлористым этилом (при $100^{\circ}$ ) [2], в то время как $\mathrm{R}_{3} \mathrm{Al}$ с ним не реагирует.

При нзучении зтой реакции на ряде примеров (см. табл.) мы установили, что скорость взаимодействия алкилгалогенидов $c \mathrm{R}_{3} \mathrm{Al}$ в дальнейшем нарастает в последовательности: $\mathrm{C}_{7} \mathrm{H}_{15} \mathrm{Cl}<\mathrm{C}_{9} \mathrm{H}_{19} \mathrm{Cl}<$ трет- $\mathrm{C}_{4} \mathrm{H}_{9} \mathrm{Cl}<\mathrm{CH}_{2}=\mathrm{CH}-\mathrm{CH}_{2} \mathrm{Cl}<\mathrm{C}_{6} \mathrm{H}_{5} \mathrm{CH}_{2} \mathrm{Cl}<$ $\left(\mathrm{C}_{6} \mathrm{H}_{5}\right)_{3} \mathrm{CCl}$.

Помимо этого, образование в ходе реакции $\mathrm{R}_{2} \mathrm{AlCl}, \mathrm{RAlCl}_{2}$ и $\mathrm{AlCl}_{3}$ обусловливает возможность протекания реакции Фриделя-Крафтса как с ароматическим растворнтелем (если он присутствует), так и с самим галоидным соединением, если в нем содержатся ароматические ядра или есть возможность легкого отрыва водорода.

\section{Экспериментальная часть}

Все использованные реактивы после тщательной очистки и высушивания по своим жонстантам соответствовали данным литературы. Технический $\left(\text { изо- } \mathrm{C}_{4} \mathrm{H}_{9}\right)_{3} \mathrm{Al}$ очищали вымораживанием' из бензина - «калоша» и чистого гептана, перегоняли в вакууме (т. кип $52-56^{\circ} / 2-3$ мм рт. ст.). Для опытов использовали 1 M раствор $\left(u з о-\mathrm{C}_{4} \mathrm{H}_{9}\right)_{3} \AA$ l в гептане; концентрацию раствора проверяли по анализу $\mathrm{Al}\left[{ }^{4}\right]$ и по объему изобутана, выделившегося при гидролизе.

Реакции алкилгалогенидов с $\left(\text { изо- } \mathrm{C}_{4} \mathrm{H}_{9}\right)_{3} \mathrm{Al}$ проводились в трубке Шленка в ат. мосфере сухого аргона без растворителя (алкилгалогениды до $\mathrm{C}_{4}$ ) или в растворе (в гептане и в бензоле). Глубину превращения $\mathrm{R}_{3} \mathrm{Al}$ в хлориды устанавливали волюмометрически, измеряя объем изобутана, выделившегося при гидролизе.

Состав выделяющихся газов определяли хроматографически, $\mathrm{HCl}$ улавливали пропусканием газа через ловушки с титрованным 1 н. раствором $\mathrm{KOH}$. После окончания реакции реакционную смесь разлагали водой. Конечные продукты были идентифицированы по элементарному анализу, температуре кипения (плавления), молекулярному весу и инфракрасным спектрам. В тех случаях, когда скорость реакции была умеренной, дополнительным «индикатором» на обмен $\mathrm{R}$ при $\mathrm{Al}$ на $\mathrm{Cl}$ служил установленный нами факт полимеризации циклопентадиена в присутствии соединений со связью $\mathrm{Al}-\mathrm{Cl}$, в то время как $\mathrm{R}_{3} \mathrm{Al}$ не полимеризует циклопентадиен. В реакционную смесь добавляли несколько миллилитров циклопентадиена; с появлением связей $\mathrm{Al}-\mathrm{Cl}$ образовывался нерастворимый полимер $\left(\mathrm{C}_{5} \mathrm{H}_{6}\right)_{x}$. 


\section{Взаимодействие алкилгалогенидов с $\left(\text { изо- } \mathrm{C}_{4} \mathrm{H}_{9}\right)_{3} \mathrm{Al}$}

Низшие алкилгалогениды, содержащие в цепи до 4 атомов углерода, реагируют с (изо- $\left.\mathrm{C}_{4} \mathrm{H}_{9}\right)_{3} \mathrm{Al}$ крайне медленно. Доказательством некоторого обмена $\mathrm{R}$ на $\mathrm{Cl}$ является образование твердого полициклопентадиена по истечении $10-12$ иас.

Алкилгалогениды с более длинной цепью или имеющие изостроение быстро и количественно реагируют с $\left(\text { изо- } \mathrm{C}_{4} \mathrm{H}_{9}\right)_{3} \mathrm{Al}$. При соотношении $\mathrm{R}^{\prime} \mathrm{Cl}: \mathrm{R}_{3} \mathrm{Al}=3: 1$ реакция ндет до сбразования $\mathrm{AlCl}_{3}$. Выделяются изобутан и изобутилен (содержание изобути лена в газе несколько ниже, чем изобутана, поскольку имеет место некоторая полимеризация изобутилена на образующихся в ходе реакции катионных катализаторах, содержащих связи $\mathrm{Al}-\mathrm{Cl}$ ), алкильные радикалы галогенида отчасти конденсируются, отчасти присоединяют $\mathrm{H}^{-}$с образованием соответствующих углеводородов. В бензоле имеет место реакция алкилирования растворителя алкильной группой галогенида, выделение $\mathrm{HCl}$ и полное разложение $\left(\text { изо- } \mathrm{C}_{4} \mathrm{H}_{9}\right)_{3} \mathrm{Al}$.

\section{Реакции хлористого аллила и хлористого бензила с $\left(\text { изо- } \mathrm{C}_{4} \mathrm{H}_{9}\right)_{3} \mathrm{AI}$}

Реакция (изо- $\left.\mathrm{C}_{4} \mathrm{H}_{9}\right)_{3} \mathrm{Al}$ с хлористым аллилом и бензилхлоридом в гептане протекает в две стадии. На первой стадии происходит вытеснение изобутильных групп у Al хлором, сопровождаюшееся выделением изобутана и изобутилена. При соотношении $\mathrm{R}^{\prime} \mathrm{X}: \mathrm{R}_{3} \mathrm{Al}=3: 1$ образуется $\mathrm{AlCl}_{3}$ (белая взвесь). Во второй стадии в случае хлористого аллила образуется смола, реакция сопровождается выделением НС1. B случае бензилхлорида во второй стадии, примерно через 20 мин, имеет место образование ярко-красного кристаллического продукта, сопровождаюшееся также интенсивным выделением $\mathrm{HCl}$. Количество $\mathrm{HCl}$ состветствует 20-30\% хлора, содержащегося в бензилхлориде (нужно иметь в виду, что часть $\mathrm{HCl}$ может растворяться в реакционной смеси). Продукт чрезвычайно чувствителен к окислению и гидролизу; по данным элементарного анализа он имеет брутто-формулу $\mathrm{Al}_{1} \mathrm{Cl}_{2} \mathrm{C}_{21} \mathrm{H}_{12}$.

При проведении этой же реакции в среде бензола взаимодействие также протекает в две стадии, которые в общем виде таковы. Первая стадия:

$$
\begin{gathered}
3 \mathrm{C}_{6} \mathrm{H}_{5} \mathrm{CH}_{2} \mathrm{Cl}+\left(u 30-\mathrm{C}_{4} \mathrm{H}_{9}\right)_{3} \mathrm{Al}+3 \mathrm{C}_{6} \mathrm{H}_{6} \rightarrow \\
\rightarrow \mathrm{AlCl}_{3}+1,5 u 30-\mathrm{C}_{4} \mathrm{H}_{10}+1,5 u 30-\mathrm{C}_{4} \mathrm{H}_{8}+3 \mathrm{C}_{6} \mathrm{H}_{5} \mathrm{CH}_{2} \mathrm{C}_{6} \mathrm{H}_{5} .
\end{gathered}
$$

Вторая стадия представляет собой реакцию Фриделя-Крафтса:

$$
\begin{aligned}
\mathrm{C}_{6} \mathrm{H}_{5} \mathrm{CH}_{2} \mathrm{Cl}+\mathrm{C}_{6} \mathrm{H}_{6} \stackrel{\mathrm{AlCl}_{3}}{\longrightarrow} \mathrm{C}_{6} \mathrm{H}_{5} \mathrm{CH}_{2} \mathrm{C}_{6} \mathrm{H}_{5}+\mathrm{HCl} \\
2 \mathrm{C}_{6} \mathrm{H}_{5} \mathrm{CH}_{2} \mathrm{Cl} \stackrel{\mathrm{AlCl}_{3}}{\longrightarrow} \mathrm{C}_{6} \mathrm{H}_{5} \overbrace{\mathrm{CH}_{2}}^{\mathrm{CH}_{2}} \mathrm{C}_{6} \mathrm{H}_{5}+\mathrm{HCl}
\end{aligned}
$$

Из реакционной смеси после гидролиза выделен дифенилметан (т. киі. '02-103\%) 4 мм рт. ст., т. пл. $25^{\circ}$, мол. вес 168,5$)$ и небольшое количество антрацена.

\section{Взаимодействие третичных алкилгалогенидов с $\left(\text { изо- } \mathrm{C}_{4} \mathrm{H}_{9}\right)_{3} \mathrm{Al}$}

$\mathrm{C}$ третичными алкилгалогенидами $\left(\mathrm{R}_{3} \mathrm{C}^{+} \mathrm{Cl}^{-}\right)$проходит очень быстрый обмен $\mathrm{R}$ на $\mathrm{Cl}$ при Al. Реакция Фриделя-Крафтса не имеет места даже в присутствии арома. тических углеводородов - очевидно, из-за пространственных препятствий; как в гегтане, так и в бензоле выделения $\mathrm{HCl}$ нет. Продукт реакции имеет ярко-желтую окраску (комплекс $\mathrm{R}_{3} \mathrm{CH} \cdot \mathrm{AlCl}_{3}$ ). После гидролиза продуктов реакций, ироведенных трифенилметилхлоридом, выделены трифенилметан (т. пл. 92\%, мол. вес 245) и небольшое количество более сложных продуктов конденсации. 
Вза имодейст ви е $\left(\text { изо- } \mathrm{C}_{4} \mathrm{H}_{9}\right)_{3} \mathrm{Al}$

\begin{tabular}{|c|c|c|c|c|c|}
\hline \multirow[b]{2}{*}{ Соединение } & \multicolumn{5}{|c|}{ В гептане } \\
\hline & $\begin{array}{l}\text { Время } \\
\text { реакций, } \\
\text { мин }\end{array}$ & $\begin{array}{c}\text { Моляр- } \\
\text { ное } \\
\text { соотно- } \\
\text { шение } \\
\mathrm{R}^{\prime} \mathrm{Cl} \\
\text { : } \mathrm{R}_{3} \mathrm{Al}\end{array}$ & $\begin{array}{c}\text { Степень } \\
\text { замещения } \\
\mathrm{R} \text { на } \mathrm{Cl} \\
\underset{\text { при } \mathrm{Al}}{\%}\end{array}$ & $\begin{array}{l}\text { Выде- } \\
\text { ление } \\
\mathrm{HCl}\end{array}$ & Состав газов \\
\hline$n-\mathrm{C}_{7} \mathrm{H}_{15} \mathrm{Cl}$ & 15 & $\begin{array}{l}1: 1 \\
3: 1\end{array}$ & $\begin{array}{r}90 \\
100\end{array}$ & - & $u 30-\mathrm{C}_{4} \mathrm{H}_{8} 40$ \\
\hline$n-\mathrm{C}_{9} \mathrm{H}_{19} \mathrm{Cl}$ & 12 & $\begin{array}{l}1: 1 \\
3: 1\end{array}$ & $\begin{array}{r}96 \\
100\end{array}$ & - & $u 30-\mathrm{C}_{4} \mathrm{H}_{10} \quad 60$ \\
\hline трет- $\mathrm{C}_{4} \mathrm{H}_{9} \mathrm{Cl}$ & 10 & $1: 1$ & 92 & - & $\begin{array}{ll}u 30-\mathrm{C}_{4} \mathrm{H}_{8} & 70 \\
u 30-\mathrm{C}_{4} \mathrm{H}_{10} & 30\end{array}$ \\
\hline $\mathrm{CH}_{2}=\mathrm{CH}-\mathrm{CH}_{2} \mathrm{Cl}$ & $\begin{array}{l}10 \\
20\end{array}$ & $\begin{array}{l}1: 1 \\
3: 1\end{array}$ & $\begin{array}{l}100 \\
100\end{array}$ & да & $\begin{array}{l}u 30-\mathrm{C}_{4} \mathrm{H}_{8} 20 \\
u 30-\mathrm{C}_{4} \mathrm{H}_{10} \quad 80\end{array}$ \\
\hline $\mathrm{C}_{6} \mathrm{H}_{5} \mathrm{CH}_{2} \mathrm{Cl}$ & 20 & $3: 1$ & 100 & да & $\begin{array}{ll}u 30-\mathrm{C}_{4} \mathrm{H}_{8} & 40 \\
u 30-\mathrm{C}_{4} \mathrm{H}_{10} & 60\end{array}$ \\
\hline$\left(\mathrm{C}_{6} \mathrm{H}_{5}\right)_{3} \mathrm{CCl}$ & $\begin{array}{l}3 \\
5\end{array}$ & $\begin{array}{l}1: 1 \\
3: 1\end{array}$ & $\begin{array}{l}100 \\
100\end{array}$ & - & $\begin{array}{l}u 30-\mathrm{C}_{4} \mathrm{H}_{8} 80 \\
u 30-\mathrm{C}_{4} \mathrm{H}_{10} 20\end{array}$ \\
\hline
\end{tabular}

Концентрация (изо- $\left.\mathrm{C}_{4} \mathrm{H}_{9}\right)_{3} \mathrm{Al}-8$ мнолей/л, объем растворителя - 100 мл, температура реакций $20^{\circ}$.

Очевидно, в ходе реакции образуются карбониевые катионы $\mathrm{R}_{3} \mathrm{C}^{+}$, которые отрывают гидрид-ион от растворителя или третичного атома C изобутильной группы, поэто. му в составе газов имеется $80 \%$ изобутилена и только до $20 \%$ изобутана.

Во всех вышеуказанных случаях, если взаимодействие $\mathrm{R}^{\prime} \mathrm{Cl}$ с $\mathrm{R}_{3} \mathrm{Al}$ проводили в ароматическом растворителе и в систему подавали этилен, протекала реакция алкилирования по Фриделі-Крафтсу. Из продуктов реакции, проводившейся в среде толуола в присутствии этилена, выделены орто-, пара- и мета-этилтолуолы (полосы в УФ-спектре при 2650, 2680, 2720, 2735 А)

\section{O природе реакции алкилгалогенидов с $\mathbf{R}_{3} \mathbf{A l}$}

При последсвательном замещении радикалов у алюминия на хлор ( $\mathrm{R}_{3} \mathrm{Al}, \mathrm{R}_{2} \mathrm{AlCl}$, $\mathrm{RAlCl}_{2}$ ) прочность связи $\mathrm{R}-\mathrm{Al}$ увеличивается: в реакциях гидролиза, окисления и т. д. второй и третий радикалы у $\mathrm{Al}$ реагируют гораздо труднее, чем первый. $\mathrm{B}$ то же время скорость реакции $\mathrm{R}_{3} \mathrm{Al} \stackrel{\mathrm{R}^{\prime} \mathrm{Cl}}{\longrightarrow} \mathrm{R}_{2} \mathrm{AlCl} \stackrel{\mathrm{R}^{\prime} \mathrm{Cl}}{\longrightarrow} \mathrm{RAlCl}_{2} \stackrel{\mathrm{R}^{\prime} \mathrm{Cl}}{\longrightarrow} \mathrm{AlCl}_{3}$ возрастает, наоборот, слева направо, т. е. по мере увеличения электрофильности молекулы соединения алюминия. Таким образом, скорость реакции обмена $\mathrm{R}$ на $\mathrm{Cl}$ при $\mathrm{Al}$ зависит от электрофильности молекулы алюминийорганического соединения в целом, а не от характера

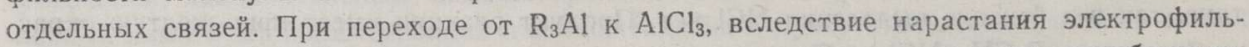
ности и полярности молекулы, увеличивается и ее склонность к комплексообразованию. Поскольку скорость взаимодействия алкилгалогенидов с $\mathrm{R}_{3} \mathrm{Al}$ возрастает с облег-

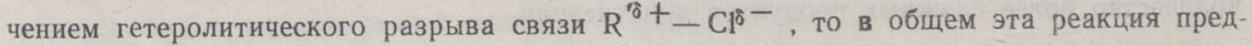




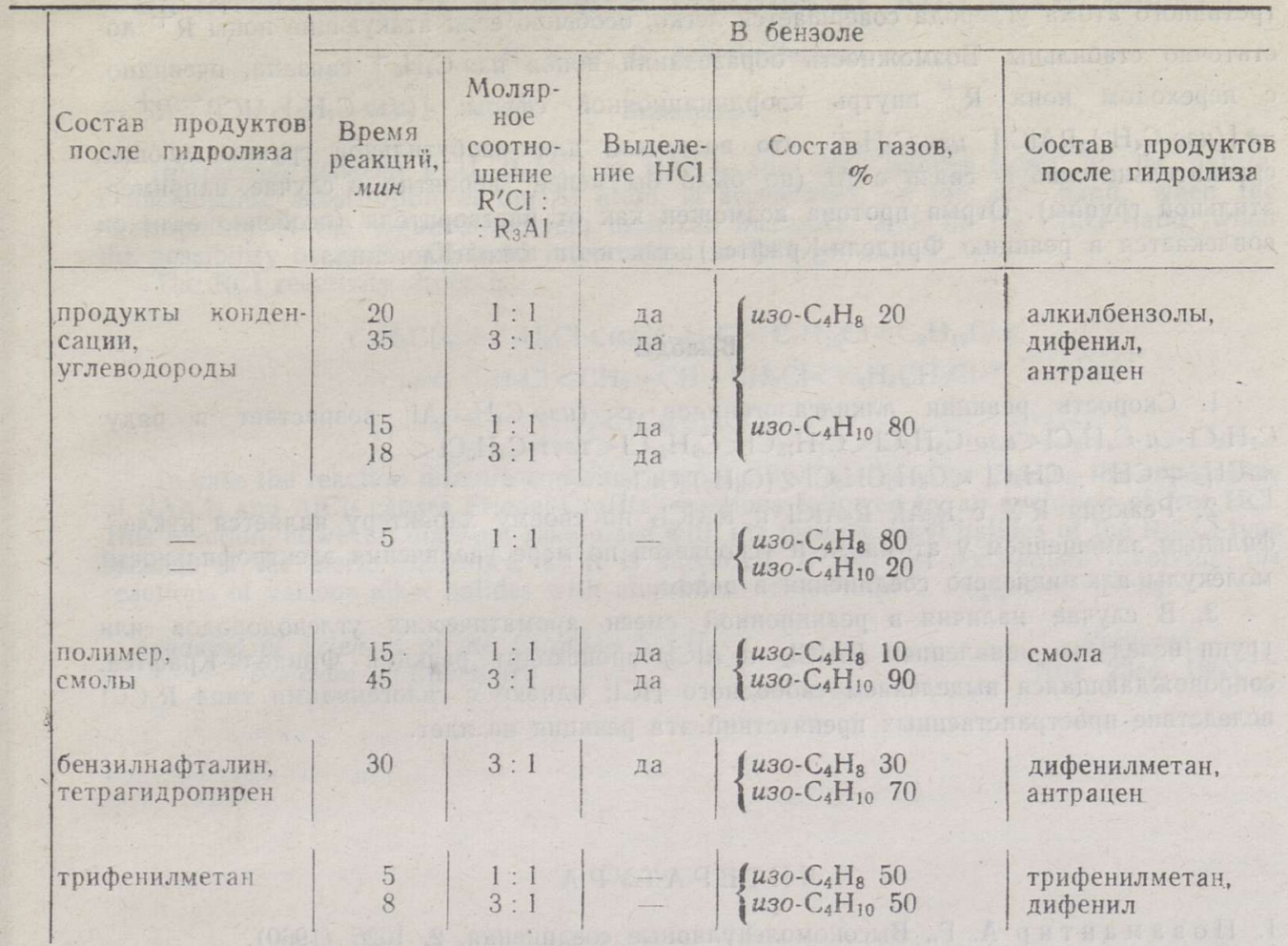

ставляет собой нуклеофильное замешение у атома $\mathrm{Al}$, проходящее через стадию комплексообразования $\left(\mathrm{R}^{\prime}+\left[\mathrm{AlR} \mathrm{R}_{3}\right]^{-}\right)$. На ионный характер реакции указывает и факт, что ни в одном случае мы не нашли в реакционной смеси продуктов рекомбинации радикалов; а при проведении реакции в резонаторе $Э П Р$, даже в случае $\mathrm{C}_{6} \mathrm{H}_{5} \mathrm{CH}_{2} \mathrm{Cl}$, $\left(\mathrm{C}_{6} \mathrm{H}_{5}\right)_{3} \mathrm{CCl}$, дающих достаточно стабнльные свободные радикалы, появления радика лов не наблюдалось.

Так как образующиеся в ходе реакции $\mathrm{R}_{2} \mathrm{AlCl}$ и $\mathrm{RAlCl}_{2}$ взаимодействуют $\mathrm{c} \mathrm{R}^{\prime} \mathrm{Cl}$ значительно быстрее, чем исходный $\mathrm{R}_{3} \mathrm{Al}$, тө реакция должна протекать с самоускорением.

Механизм взаимодействия (изо- $\left.\mathrm{C}_{4} \mathrm{H}_{9}\right)_{3} \mathrm{AI}$ и $\mathrm{R}^{\prime} \mathrm{Cl}$ мы изобразим для простоты в виде ряда ионных реакций, хотя в большинстве из них участвуют скорее не свободные ионы, а ионы, входящие в состав поляризованных комплексов.

1. $\left(\text { изо }-\mathrm{C}_{4} \mathrm{H}_{9}\right)_{3} \mathrm{Al}+\mathrm{RCl} \rightarrow\left[\left(\text { изo- } \mathrm{C}_{4} \mathrm{H}_{9}\right)_{3} \mathrm{AlCl}\right]-\mathrm{R}^{+} \gtrless\left(u 30-\mathrm{C}_{4} \mathrm{H}_{9}\right)_{2} \mathrm{AlCl}+u 3 o-\mathrm{C}_{4} \mathrm{H}_{9}-+\mathrm{R}^{+}$

$$
\underset{\rightarrow}{\rightarrow} u 30 \cdot \mathrm{C}_{4} \mathrm{H}_{8}+\mathrm{RH}
$$

2. $u 30 \cdot \mathrm{C}_{4} \mathrm{H}_{9}^{-} \stackrel{430 \cdot \mathrm{C}_{4} \mathrm{H}_{9}^{+}}{\longrightarrow} u 30-\mathrm{C}_{4} \mathrm{H}_{8}+u 30 \cdot \mathrm{C}_{4} \mathrm{H}_{10}$

$$
\underset{\longrightarrow}{\longrightarrow} \text { uso- } \mathrm{C}_{4} \mathrm{H}_{10}
$$

Относительные количества изобутана и изобутилена зависят от имеющихся в системе возможностей для протекания реакции (2) в том или нном из указанных направ. 
лений. Ион $\mathrm{R}^{+}$не присоединяет $и з \mathrm{C}_{4} \mathrm{H}_{9}^{-}$, а отрывает от него $\mathrm{H}^{-}$. Такой отрыв от третичного атома углерода совершается легко, особенно если атакующие ионы $\mathrm{R}^{+}$достаточно стабильны. Возможность образования ионов изо- $\mathrm{C}_{4} \mathrm{H}_{9}+$ связана, очевидно, с переходом иона $\mathrm{R}^{+}$внутрь координационной сферы: $\left[\left(\text {изо }^{-} \mathrm{C}_{4} \mathrm{H}_{9}\right)_{3} \mathrm{AlCl}\right]^{-} R^{+} \rightarrow$ $\rightarrow\left[\left(\text { изо- } \mathrm{C}_{4} \mathrm{H}_{9}\right){ }_{2} \mathrm{RAICI}\right]^{-}$изо- $\mathrm{C}_{4} \mathrm{H}_{9}{ }^{+}$, что возможно для изобутильной группы, дающей еравнительно слабую связь с Al (но было бы менее вероятно в случае, например, этильной группы). Отрыв протона возможен как от растворителя (особенно если он вовлекается в реакцию Фриделя-Крафтса), так н от алкилгалогенида.

\section{Выводы}

1. Скорость реакции алкилгалогенидов с $\left(\text { изо- } \mathrm{C}_{4} \mathrm{H}_{9}\right)_{3} \mathrm{Al}$ возрастает в ряду $\mathrm{C}_{2} \mathrm{H}_{5} \mathrm{Cl}<n-\mathrm{C}_{4} \mathrm{H}_{9} \mathrm{Cl}<$ uso- $\mathrm{C}_{3} \mathrm{H}_{7} \mathrm{Cl}<\mathrm{C}_{7} \mathrm{H}_{15} \mathrm{Cl}<\mathrm{C}_{9} \mathrm{H}_{19} \mathrm{Cl}<$ tper $-\mathrm{C}_{4} \mathrm{H}_{9} \mathrm{Cl}<$ $<\mathrm{CH}_{2}=\mathrm{CH}-\mathrm{CH}_{2} \mathrm{Cl}<\mathrm{C}_{6} \mathrm{H}_{5} \mathrm{CH}_{2} \mathrm{Cl}<\left(\mathrm{C}_{6} \mathrm{H}_{5}\right)_{3} \mathrm{CCl}$.

2. Реакция $\mathrm{R}^{\prime} \mathrm{X}$ с $\mathrm{R}_{3} \mathrm{Al}, \mathrm{R}_{2} \mathrm{AlCl}$ и $\mathrm{RAlCl}_{2}$ по своему характеру является нуклеофильным замещением у атома $\mathrm{Al}$ и ускоряется по мере увеличения электрофильности молекулы алюминиевого соединения в целом.

3. В случае наличия в реакционной смеси ароматических углеводородов или групп вслед за появлением $\mathrm{RAlCl}_{2}$ и $\mathrm{AlCl}_{3}$ происходит реакция Фриделя-Крафтса, сопровождающаяся выделением свободного $\mathrm{HCl}$; однако с галогенидами типа $\mathrm{R}_{3} \mathrm{CC}$ ! вследствие-пространственных препятствий эта реакция не идет.

\section{ЛИТЕ Р А Т У Р А}

1. По зам анти р А. Г., Высокомолекулярные соединения, 2, 1026 (1960).

2. Поз а м н ти р А. Г., Г ен усов М. П., ЖОХ, вып. $4,32,1175$ (1962).

3. Z i egle r K., cm. «Örganometallic Chemistry», ACS Monograph № 147, ed. by H. Zeiss, New York, 1960, стр. 198.

4. Houd a M., Körbl J., B a ž a n t V., Př ib y 1 R., Chem. Listy, 51, 2259 (1957).
Ннститут химии
Академии наук Эстонской ССР
Поступила в редакцию
23. XI 1963

\section{TRI-ISOBUTUULALUMIINIUMI REAKTSIOONIDEST ALKUULHALOGENIIDIDEGA}

\section{H. Kaar, G. Schwindlerman}

\section{Resümee}

$\mathrm{R}_{3} \mathrm{Al}, \mathrm{R}_{2} \mathrm{AlCl}$ ja $\mathrm{RAlCl}_{2}$ reaktsioonid alküülhalogeniididega on oma iseloomult alumiiniumi aatomiga seotud alküülgruppide järkjärguline nukleofiilne asendumine halogeeniga.

Reaktsioon kiireneb ühelt poolt alumiiniumorgaanilise ühendi molekuli kui terviku elektrofiilsuse suurenemisega, teiselt poolt R・ga seotud kloori eraldumise kiiruse kasvuga reas

$$
\begin{gathered}
\mathrm{C}_{2} \mathrm{H}_{5} \mathrm{Cl}<n-\mathrm{C}_{4} \mathrm{H}_{9} \mathrm{Cl}<\text { iso- } \mathrm{C}_{3} \mathrm{H}_{7} \mathrm{Cl}<\mathrm{C}_{7} \mathrm{H}_{15} \mathrm{Cl}<\mathrm{C}_{9} \mathrm{H}_{19} \mathrm{Cl}< \\
<\text { terts } \mathrm{C}_{4} \mathrm{H}_{9} \mathrm{Cl}<\mathrm{CH}_{2}=\mathrm{CH}-\mathrm{CH}_{2} \mathrm{Cl}<\mathrm{C}_{6} \mathrm{H}_{5} \mathrm{CH}_{2} \mathrm{Cl}<\left(\mathrm{C}_{6} \mathrm{H}_{5}\right)_{3} \mathrm{CCl} \text {. }
\end{gathered}
$$

Kui reaktsioonisegu sisaldab aromaatseid süsivesinikke või gruppe, kutsub $\mathrm{RAICl}_{2}$ ja $\mathrm{AlCl}_{3}$ tekkimine esile Friedel-Craffts'i reaktsiooni vaba $\mathrm{HCl}$ eraldumisega. See reaktsioon ei toimu tertsiaarsete alküülhalogeniidide $\left(\mathrm{R}_{3} \mathrm{CCl}\right)$ puhul ruumiliste takistuste tõttu.

Artiklis esitatakse mitmesuguste alküülhalogeniidide ja tri-isobutüülalumiiniumi reaktsioonide mehhanismid. 


\section{ON INTERACTION OF ALUMINIUM TRI-ISOBUTYL WITH ALKYL-HALIDES}

\section{H. Kaar, G. Schwindlerman}

\section{Summary}

Interaction between $\mathrm{R}_{3} \mathrm{Al}, \mathrm{R}_{2} \mathrm{AlCl}$ or $\mathrm{RAlCl}_{2}$ and alkyl halides being, by its nature, a nucleophilic substitution at the Al atom, is accelerated, on the one hand. when the electrophility of the organoaluminium molecule increases, and,- on the other hand, when the possibility of an $\mathrm{C}-\mathrm{Cl}$ bond interruption in $\mathrm{RCl}$ grows.

The $\mathrm{RCl}$ reactivity order is:

$$
\begin{gathered}
\mathrm{C}_{2} \mathrm{H}_{5} \mathrm{Cl}<n-\mathrm{C}_{4} \mathrm{H}_{9} \mathrm{Cl}<\text { ise }-\mathrm{C}_{3} \mathrm{H}_{7} \mathrm{Cl}<\mathrm{C}_{7} \mathrm{H}_{15} \mathrm{Cl}<\mathrm{C}_{9} \mathrm{H}_{19} \mathrm{Cl}< \\
<\text { tert. } \mathrm{C}_{4} \mathrm{H}_{9} \mathrm{Cl}<\mathrm{CH}_{2}=\mathrm{CH}-\mathrm{CH}_{2} \mathrm{Cl}<\mathrm{C}_{6} \mathrm{H}_{5} \mathrm{CH}_{2} \mathrm{Cl}< \\
<\left(\mathrm{C}_{6} \mathrm{H}_{5}\right)_{3} \mathrm{CCl} .
\end{gathered}
$$

In case the reaction mixture contains aromatic hydrocarbons or groups, the appearance of $\mathrm{RAICl}_{2}$ and $\mathrm{AlCl}_{3}$ causes Friedel-Craffts' reactions followed by an evolution of free $\mathrm{HCl}$. This reaction, however, does not take place with the tertiary alkyl halides of the $\mathrm{R}_{3} \mathrm{CCl}$-type because of the steric hindrance, as it is supposed. A reaction mechanism involving the reactions of various alkyl halides with aluminium tri-isobutyl is suggested hereby. Academy of Sciences of the Estonian S.S.R.,
Institute of Chemistry
Received Nov. 23rd, 1963 